\title{
Participación en sociedades científicas estudiantiles y en cursos extracurriculares de investigación, asociados a la producción científica de estudiantes de medicina humana: estudio preliminar
}

\author{
Carlos J. Toro-Huamanchumo, Virgilio E. Failoc-Rojas, Cristian Díaz-Vélez
}

Introducción. La promoción de la investigación científica durante el pregrado representa una pieza fundamental para garantizar una educación médica continua y una formación integral del estudiante, debiendo ser una prioridad en todas las universidades.

Objetivo. Identificar los factores que podrían estar asociados con la producción científica del estudiante de medicina humana de una región del Perú.

Sujetos y métodos. Estudio analítico transversal. Los alumnos respondieron una encuesta autoadministrada, donde se les pidió información sobre su producción científica, asistencia a cursos extracurriculares de investigación, experiencia y motivos para hacer investigación. Se cruzaron las variables: pertenecer a una sociedad científica, realizar cursos extracurriculares de metodología, bioestadística, lectura crítica y búsqueda bibliográfica, con el hecho de tener dos o más trabajos y dos o más publicaciones. Para el análisis inferencial se usó razón de prevalencias y la prueba de Fisher con un intervalo de confianza del 95\%. Se consideraron como significativos los valores de $p<0,05$.

Resultados. De los 50 alumnos encuestados, el 44\% pertenecía a una sociedad científica, el $60 \%$ había asistido al menos a un curso extracurricular y sólo el $20 \%$ tenía alguna publicación científica. Hubo asociación entre la pertenencia a una sociedad y la asistencia a un curso extracurricular con haber realizado dos o más trabajos y publicaciones.

Conclusión. Son pocos los alumnos con algún trabajo de investigación o alguna publicación. Pertenecer a sociedades científicas y participar en cursos extracurriculares de investigación aumenta la probabilidad de tener una mayor producción científica.

Palabras clave. Cursos de capacitación. Educación de pregrado en medicina. Estudiantes de medicina. Perú. Promoción de la investigación.

Participation in student scientific societies and research courses, associated with the scientific production of medical students: a preliminary study

Introduction. The promotion of scientific research within the undergraduate represents a fundamental to ensure continuous medical education and comprehensive training of the student, and should be a priority for all universities.

Aim. To identify factors that might be associated with the scientific production of medical student from a region of Peru.

Subjects and methods. An analytical, cross-sectional study was conducted. The students answered a self-administered survey where they were asked about their scientific production, their attending to extracurricular courses, their research experience and the reasons for doing research. Crossed variables: belong to a scientific society, attending to extracurricular courses in methodology, biostatistics, critical reading and literature search, with having two or more researches and two or more publications. For the inferential analysis we used the prevalence ratio and Fisher test used with a $95 \%$ confidence interval. We considered as a significant value $p<0.05$.

Results. Of the 50 students surveyed, $44 \%$ belonged to a scientific society, $60 \%$ had attended to at least one extracurricular course and only $20 \%$ had any scientific publication. There was association between belonging to a scientific society and have attended to an extracurricular course with having two or more researches and a publications.

Conclusion. There were few students with a research or a publication. Membership in scientific societies and participation in extracurricular research courses increases the probability of having a higher scientific production.

Key words. Medical students. Peru. Research promotion. Training courses. Undergraduate.
Sociedad Científica de Estudiantes de Medicina Veritas, SCIEMVE (C.J. Toro-Huamanchumo). Sociedad Científica de Estudiantes de Medicina de la Universidad Nacional Pedro Ruiz Gallo, SOCIEM-UNPRG (V.E. Failoc-Rojas). Sociedad Científica Médico Estudiantil Peruana, SOCIMEP (C.J. Toro-Huamanchumo, V.E. Failoc-Rojas). Facultad de Medicina Humana; Universidad de San Martín de Porres; Chiclayo (C.J. ToroHuamanchumo). Facultad de Medicina Humana; Universidad Nacional Pedro Ruiz Gallo; Lambayeque (V.E. Failoc-Rojas). Oficina de Inteligencia Sanitaria; Hospital Nacional Almanzor Aguinaga Asenjo; Chiclayo, Perú (C. Díaz-Vélez).

Correspondencia:

Dr. Cristian Díaz Vélez. Oficina de Inteligencia Sanitaria. Hospital Nacional Almanzor Aguinaga Asenjo. Chiclayo, Perú.

E-mail:

cristiandiazv@hotmail.com

Conflicto de intereses:

No declarado.

Competing interests: None declared.

(c) 2015 FEM 


\section{Introducción}

La promoción de la investigación científica dentro del pregrado representa una pieza fundamental para garantizar una educación médica continua y una formación integral del estudiante [1]. Por ello, la educación en una cultura de investigación debe ser una prioridad en las universidades porque el fin principal de éstas no es concebir profesionales en masa, sino generar conocimiento nuevo a través de la investigación científica [2,3]. Si una universidad no promueve el desarrollo del pensamiento crítico y la actividad científica en el estudiante, no está cumpliendo su rol esencial.

Existen deficiencias y limitaciones en las iniciativas tomadas por parte de las universidades latinoamericanas para el desarrollo de eventos que promuevan el espíritu científico y permitan mejorar las habilidades en investigación de los estudiantes [1]. Experiencias en España han demostrado que la organización de estos eventos tiene un valor formativo importante para el alumno, el docente y la universidad misma [4].

Por suerte, en Perú existen las sociedades científicas de estudiantes de medicina (SOCEM), agrupaciones estudiantiles sin fines de lucro y apartidistas, que tienen como finalidad promover la investigación durante el pregrado [5]. Actualmente, la integración de dichas sociedades da cabida a la Sociedad Científica Médico Estudiantil Peruana (SOCIMEP), cuyo fin es implementar mecanismos que permitan elevar el nivel científico-académico de los estudiantes de medicina en Perú, promoviendo e incentivando la investigación biomédica desde el pregrado [6].

No obstante, existen casos en los cuales hay falta de conocimiento por parte de las autoridades universitarias acerca de la importancia, necesidad e impacto que tienen las SOCEM en la producción científica nacional, tanto a corto como a largo plazo [2].

Es importante tener en cuenta que en ellas se genera un ambiente propicio para el desarrollo y perfeccionamiento de los conocimientos, actitudes y prácticas del estudiante hacia la investigación [3,7]. Las SOCEM son el lugar donde se establecen conexiones estudiante-estudiante y estudiante-asesor, se dan los primeros pasos en la conformación de redes de colaboración, pilar importante en el incremento de la producción científica de las instituciones nacionales [8], y se suplementa la formación universitaria mediante la realización de cursos extracurriculares y programas de capacitación en búsqueda bibliográfica, lectura crítica, metodología de la investigación, bioestadística, redacción científica y publicación $[1,9]$.
Por lo ya mencionado, y dado que el adecuado desenvolvimiento en el ámbito científico durante el pregrado será la base y punto de partida de las investigaciones que se realicen en la etapa profesional, se plantearon como objetivos del estudio identificar los factores que podrían estar asociados con la producción científica del estudiante de medicina humana de una región del Perú, describir la experiencia que tienen en el ámbito científico y mencionar sus principales motivos para hacer investigación.

\section{Sujetos y métodos}

El presente es un estudio transversal analítico, realizado en los estudiantes de medicina humana que participaron en un curso de metodología de la investigación organizado por la Sociedad Científica de Estudiantes de Medicina de la Universidad Nacional Pedro Ruiz Gallo (SOCIEM-UNPRG) en septiembre de 2013.

Se aplicó un cuestionario validado por expertos (Tabla I) en el cual se explicaban los motivos y propósitos del estudio. El instrumento constó de tres partes:

- Edad, sexo, año de estudios y pertenencia o no a una SOCEM.

- Experiencia en el área científica [1], expresada en el número de trabajos de investigación, proyectos científicos actuales y publicaciones en revistas indizadas, y en la asistencia a cursos extracurriculares previos relacionados con la investigación científica.

- Motivación que tuvo el estudiante para realizar investigación y percepción acerca de la importancia de investigar.

Para el análisis de datos se usaron los programas SPSS v. 19.0 y Epidat v. 3.1. En el análisis bivariado se cruzaron las variables: pertenecer a una SOCEM, realizar cursos extracurriculares de metodología, bioestadística, lectura crítica y búsqueda bibliográfica, con el hecho de tener dos o más trabajos y dos o más publicaciones. Para la asociación entre las variables y la producción científica se utilizó la razón de prevalencias (RP), con un intervalo de confianza del 95\% (IC 95\%), considerando como significativos los valores de $p<0,05$.

\section{Resultados}

Se encuestó a todos los alumnos que participaron en el curso de metodología de investigación y se en- 
contraban entre el primer y sexto año de la carrera médica $(n=50)$, con un porcentaje de respuesta del $100 \%$. La mediana de edad fue de 22 años, y el rango, de 17-26 años. De los encuestados, 32 eran varones (64\%). El 52\% y $16 \%$ de los estudiantes estaban en cuarto y quinto año, respectivamente. Primero, segundo, tercero, cuarto y sexto año tuvieron sólo cuatro alumnos cada uno (8\%), y del total de encuestados, sólo 22 pertenecían a una SOCEM (44\%).

Los cursos realizados extracurricularmente con mayor asistencia fueron: búsqueda bibliográfica (50\%) y metodología de la investigación (42\%), seguido de lectura crítica (26\%) y bioestadística (20\%). El 40\% nunca había asistido a un curso extracurricular relacionado con la investigación y sólo el 26\%, 30\% y $10 \%$ de estudiantes tenían al menos dos proyectos, trabajos y publicaciones científicas, respectivamente.

Existía una mayor producción científica en quienes pertenecían a una SOCEM. Además, los estudiantes que no habían asistido nunca a algún curso de metodología de la investigación, bioestadística, lectura crítica o búsqueda bibliográfica presentaron una producción científica baja (Tabla II).

La pertenencia a una SOCEM se asoció significativamente con tener una mayor producción científica, tanto en trabajos (RP: 2,8; IC 95\%: 1,55-5) como en publicaciones (RP: 2,41; IC 95\%: 1,55-3,74). Lo mismo sucedió con la asistencia a cursos de metodología de la investigación, bioestadística, lectura crítica y búsqueda bibliográfica (Tabla III).

Por otro lado, el 44,8\% de los estudiantes indicó que la motivación para hacer investigación había nacido de uno mismo. El $34,5 \%$ sostuvo que no hubo motivación alguna; por el contrario, la realización del trabajo de investigación se encontraba dentro de la exigencia académica de alguno de los cursos de su universidad, es decir, fue obligatorio.

Finalmente, el $62 \%$ de estudiantes consideraba importante investigar porque se forma en la persona una cultura de investigación y un $28 \%$ cree que su importancia radica en la contribución a la producción científica nacional (Tabla IV).

\section{Discusión}

Uno de los aspectos destacados del estudio es el criterio que se usó para definir la producción científica del estudiante. Dado que un solo trabajo o publicación no son necesariamente sinónimos de una adecuada producción científica, se definió como tal a aquella que implique el haber realizado al menos dos trabajos de investigación o dos publicaciones en revistas indizadas.
Tabla I. Cuestionario.

Edad (años):
Sexo (masculino / femenino)
Año de estudios:
Pertenece a una sociedad científica (sí / no)
1. Mencione si ha realizado anteriormente de manera extracurricular
algún curso relacionado con la investigación
Metodología de la investigación (sí / no)
Estadística en salud (sí / no)
Lectura crítica (sí / no)
Búsqueda bibliográfica (sí / no)

2. Número de trabajos de investigación que ha realizado:

3. Número de proyectos científicos que ha realizado:

4. Número de publicaciones que tiene en revistas indizadas:

5. Responder únicamente si en las preguntas 2,3 y 4 su respuesta fue diferente de cero: ¿quién le motivó a realizar los trabajos, proyectos, publicaciones? (puede marcar más de una):

Motivación propia

Motivación por parte del autor principal

Motivación por parte de algún asesor o docente

No hubo motivación, fue obligatorio en algún curso (incluye

hacer tesis de graduación)

6. ¿Cree que es importante hacer investigación?, ¿por qué? (escoja sólo una respuesta):

Sí, porque contribuyo a la producción científica nacional

Sí, porque se forma en la persona una cultura de investigación

Sí, porque da un mayor peso al currículo

Sí, porque me da mayor reconocimiento en el mundo científico

No, porque no aporta nuevos conocimientos

No, porque es una pérdida de tiempo

No, porque hay otras cosas más importantes en medicina Otros:

En el estudio se observó que la mayoría de alumnos que se inscribieron al curso eran de cuarto y quinto año, lo cual puede deberse a que ya han seguido previamente cursos de epidemiología, salud pública o metodología de la investigación dentro del plan de estudios de su universidad y se han sentido atraídos por el ámbito científico; o tal vez que, al ver cercano el momento de elaborar su proyecto de tesis, consideran importante afianzar los conocimientos en investigación. Quizá por estos motivos es que, a partir del cuarto año, los estudiantes suelen tener un mejor conocimiento sobre la investigación, como señala un estudio previo [3]. 
Tabla II. Proyectos, trabajos y publicaciones científicas de los estudiantes de medicina, en relación a las variables de estudio.

\begin{tabular}{|c|c|c|c|c|c|c|c|}
\hline & & \multicolumn{2}{|c|}{ Proyectos } & \multicolumn{2}{|c|}{ Trabajos } & \multicolumn{2}{|c|}{ Publicaciones } \\
\hline & & $\leq 1$ & $\geq 2$ & $\leq 1$ & $\geq 2$ & $\leq 1$ & $\geq 2$ \\
\hline \multirow{2}{*}{$\begin{array}{l}\text { Pertenencia } \\
\text { a una SOCEM }\end{array}$} & Sí & $11(22 \%)$ & $11(22 \%)$ & $10(20 \%)$ & $12(24 \%)$ & $17(34 \%)$ & $5(10 \%)$ \\
\hline & No & 26 (52\%) & $2(4 \%)$ & 25 (50\%) & $3(6 \%)$ & $28(56 \%)$ & 0 \\
\hline \multirow{2}{*}{$\begin{array}{l}\text { Curso de metodología } \\
\text { de la investigación }\end{array}$} & Sí & $11(22 \%)$ & $10(20 \%)$ & $8(16 \%)$ & $13(26 \%)$ & 16 (32\%) & $5(10 \%)$ \\
\hline & No & $26(52 \%)$ & $3(6 \%)$ & $27(54 \%)$ & $2(4 \%)$ & $29(58 \%)$ & 0 \\
\hline \multirow{2}{*}{$\begin{array}{l}\text { Curso de } \\
\text { bioestadística }\end{array}$} & Sí & $2(4 \%)$ & $8(16 \%)$ & $2(4 \%)$ & $8(16 \%)$ & 7 (14\%) & $3(6 \%)$ \\
\hline & No & 35 (70\%) & $5(10 \%)$ & $33(66 \%)$ & $7(14 \%)$ & $38(76 \%)$ & $2(4 \%)$ \\
\hline \multirow{2}{*}{$\begin{array}{l}\text { Curso de } \\
\text { lectura crítica }\end{array}$} & Sí & $7(14 \%)$ & $6(12 \%)$ & $5(10 \%)$ & $8(16 \%)$ & $8(16 \%)$ & $5(10 \%)$ \\
\hline & No & $30(60 \%)$ & $7(14 \%)$ & 30 (60\%) & $7(14 \%)$ & 37 (74\%) & 0 \\
\hline \multirow{2}{*}{$\begin{array}{l}\text { Curso de búsqueda } \\
\text { bibliográfica }\end{array}$} & Sí & $15(30 \%)$ & $10(20 \%)$ & $12(24 \%)$ & $13(26 \%)$ & $20(40 \%)$ & $5(10 \%)$ \\
\hline & No & 22 (44\%) & $3(6 \%)$ & 23 (46\%) & $2(4 \%)$ & 25 (50\%) & 0 \\
\hline
\end{tabular}

Tabla III. Análisis de los factores asociados a la producción científica de los estudiantes participantes del estudio $(p<0,05)$.

\begin{tabular}{lcccccc}
\hline & \multicolumn{2}{c}{$\geq 2$ trabajos } & & \multicolumn{2}{c}{$\geq 2$ publicaciones } \\
\cline { 2 - 3 } \cline { 6 - 7 } & RP & IC 95\% & & RP & IC 95\% \\
\hline Pertenencia a una SOCEM & 2,8 & $1,57-5$ & & 2,41 & $1,55-3,74$ \\
\hline Curso de metodología de la investigación & 3,79 & $2-7,2$ & & 2,56 & $1,62-4,03$ \\
\hline Curso de bioestadística & 9,34 & $2,24-38,87$ & & 3,86 & $1,44-10,36$ \\
\hline Curso de lectura crítica & 3,73 & $1,46-9,55$ & & 4,96 & $2,58-9,53$ \\
\hline Curso de búsqueda bibliográfica & 2,53 & $1,53-4,17$ & & 2,06 & $1,38-3,08$ \\
\hline
\end{tabular}

IC 95\%: intervalo de confianza al 95\%; RP: razón de probabilidades. Con la calculadora estadística Epidat v. 3.1 se sumó 0,5 a las casillas con valores de 0 , para evitar el cálculo de indeterminación.

Si bien los cursos con mayor asistencia fueron los de búsqueda bibliográfica y metodología de la investigación, un dato preocupante es que el $40 \%$ de los encuestados no había asistido nunca a alguno de los cursos mencionados. Este hecho puede deberse a la falta de tiempo, desconocimiento de la realización de estos cursos o simplemente desinterés al respecto por parte de los estudiantes, limitaciones ya conocidas en el proceso de investigación científi- ca $[1,8,10,11]$. Por este motivo, resulta necesario continuar realizando, innovando y difundiendo cada vez más estos cursos, con una mayor promoción de los de bioestadística y manejo de programas estadísticos, pues ambos son los que generan al estudiante un mayor número de dificultades $[3,8]$.

La asistencia extracurricular a un curso de metodología de la investigación aumentó en un $279 \%$ la probabilidad de tener dos o más trabajos de investigación, y en un $156 \%$, la probabilidad de tener dos o más publicaciones. La asistencia a cursos de bioestadística aumentó ocho veces más la probabilidad de tener dos o más trabajos de investigación, y en un $286 \%$, contar con dos o más publicaciones. A esto se suman situaciones similares con los cursos de lectura crítica y de búsqueda bibliográfica, lo cual concluiría en una fuerte asociación existente entre la asistencia a cursos relacionados con la investigación y la producción científica del estudiante. Como ya se mencionó, es fundamental la organización de estos cursos y que, a la vez, se busque una participación activa de los estudiantes. Aquí es donde deben entrar las SOCEM, pues es en estos grupos donde se promueve la capacitación continua y permanente en diversos temas académicos y científicos a través de cursos, talleres, charlas y asesoría constante $[12,13]$.

Por otra parte, los participantes del estudio consideraban que era importante investigar y que se forme en el estudiante una cultura de investigación. No obstante, sólo el 38\% tenía al menos un trabajo y solamente el $20 \%$ contaba con alguna publicación científica, por lo que se podría inferir que los alumnos conocen la importancia de investigar y publicar, pero carecen de las herramientas necesarias para hacerlo, las mismas que deberían ser facilitadas por las universidades.

El hecho de pertenecer a una SOCEM aumentó en un $180 \%$ la probabilidad de tener dos o más trabajos de investigación y en un $141 \%$ la de tener dos o más publicaciones científicas. Esta situación confirmaría que aquellos alumnos que pertenecen a grupos de investigación cuentan con una mayor probabilidad de tener un buen nivel de conocimientos y una adecuada actitud, además de existir asociación entre la pertenencia a estos grupos con tener algún proyecto o trabajo de investigación, como indican algunos estudios previos $[3,8]$.

Un dato que no puede pasarse por alto es que si bien existe una actitud correcta en los estudiantes de medicina, aún no hay reflejo de ello en la producción científica. Un ejemplo claro se presenta en este estudio, donde sólo 15 y 5 alumnos tenían al menos dos trabajos de investigación y publicacio- 
nes, respectivamente. Estudios previos [3,8,14] han comunicado resultados similares: una escasa producción científica estudiantil, pese a una buena actitud hacia la investigación.

La mayoría de los estudiantes indicó que la motivación para hacer investigación nació de uno mismo, lo cual representaría un grupo humano con buenas capacidades y habilidades que deben explotarse correctamente. No obstante, también hubo alumnos que revelaron no haber tenido motivación alguna, sino que fue algo obligatorio dentro de alguno de los cursos de su carga académica, lo cual podría indicar la existencia de ciertas deficiencias en la educación universitaria para una adecuada formación del estudiante en una cultura de investigación. Además, pocos alumnos refieren a algún docente como fuente de motivación. Esto se debe a que, en Lambayeque, escasos docentes se dedican a la investigación y en su mayoría pertenecen a universidades públicas [15].

Las razones por las cuales los participantes del estudio consideraban importante investigar se relacionan una con la otra: al crearse una cultura de investigación en el estudiante, se asegura una contribución a la producción científica nacional, lo cual augura, en lo individual, una excelente vida profesional y, en general, un crecimiento del país.

El estudio presenta algunas limitaciones: debido al tamaño de la población incluida no es posible hacer una extrapolación a la realidad estudiantil nacional. Así mismo, posiblemente la mayoría de estudiantes representa un público motivado por la investigación, por lo que las motivaciones y percepciones pueden ser no representativas. No obstante, la importancia del estudio radica en que funcione como un estudio preliminar para un posterior trabajo multicéntrico que aborde los factores asociados a la producción científica de los estudiantes de medicina humana de Perú, donde se utilizará una muestra representativa y un nivel de evidencia mayor.

En conclusión, la producción científica de los estudiantes es baja. La pertenencia a una sociedad científica y la participación en cursos extracurriculares de investigación aumenta la probabilidad de incrementar su aporte científico.

Recomendamos mejorar las capacitaciones ofrecidas por parte de la SOCIMEP y continuar fomentando el espíritu investigador. Del mismo modo, se sugiere que las universidades apoyen el trabajo de las SOCEM y, conjuntamente, elaboren estrategias y sistemas académicos que permitan disminuir cada vez más los pobres indicadores de la actividad científica universitaria y generen un ambiente propicio para la creación de conocimiento en sus facultades.
Tabla IV. Motivación y percepción de los estudiantes de medicina para hacer investigación.

\begin{tabular}{|c|c|c|c|}
\hline & & $n$ & $\%$ \\
\hline \multirow{4}{*}{$\begin{array}{l}\text { Qué o quién motivó } \\
\text { para realizar } \\
\text { investigación }{ }^{a, b}\end{array}$} & Motivación propia & 13 & 44,8 \\
\hline & No hubo motivación, fue obligatorio & 10 & 34,5 \\
\hline & Motivación por parte de algún asesor o docente & 6 & 20,7 \\
\hline & Motivación por parte del autor principal & 5 & 17,2 \\
\hline \multirow{4}{*}{$\begin{array}{l}\text { Por qué consideran } \\
\text { que es importante } \\
\text { investigar }\end{array}$} & Porque se forma en la persona una cultura de investigación & 31 & 62,0 \\
\hline & Porque contribuyo a la producción científica nacional & 14 & 28,0 \\
\hline & Porque me da mayor reconocimiento en el mundo científico & 3 & 6,0 \\
\hline & Porque da mayor peso a mi currículo & 2 & 4,0 \\
\hline
\end{tabular}

a Sólo respondieron quienes tenían al menos un trabajo o proyecto de investigación $(n=29)$; ${ }^{b}$ El estudiante podía elegir más de una alternativa.

\section{Bibliografía}

1. Mayta-Tristán P, Cartagena-Klein R, Pereyra-Elías R, Portillo A, Rodríguez-Morales AJ. Apreciación de estudiantes de medicina latinoamericanos sobre la capacitación universitaria en investigación científica. Rev Med Chile 2013; 141: 716-22.

2. Mayta-Tristán P. Sociedades científicas de estudiantes de medicina: el futuro de la investigación en Latinoamérica. MedUNAB 2010; 13: 3-4.

3. Cabrera-Enríquez JA, Cruzado-Mendoza C, Purizaca-Rosillo N, López-Samanamú RO, Lajo-Aurazo Y, Peña-Sánchez ER, et al. Factores asociados con el nivel de conocimientos y la actitud hacia la investigación en estudiantes de medicina en Perú, 2011. Rev Panam Salud Pública 2013; 33: 166-73.

4. Arráez-Aybar LA, Núñez-Cortés JM, Carabantes-Alarcón D, Lozano-Fernández R, Iglesias-Peinado I, Palacios-Alaiz E, et al. Adquisición de competencias transversales en alumnos de pregrado de Ciencias de la Salud en la Universidad Complutense: una experiencia positiva. Educ Med 2008; 11: 169-77.

5. Mayta-Tristán P, Peña-Oscuvilca A. Importancia de la publicación en las sociedades científicas de estudiantes de medicina del Perú: estudio preliminar. CIMEL 2009; 14: 27-34.

6. Taype-Rondán A, Lajo-Aurazo Y, Gutiérrez-Brown R, ZamalloaMasías N, Saldaña-Gonzales M. Aporte de las sociedades estudiantiles en la publicación científica en Scielo-Perú, 2009-2010. Rev Peru Med Exp Salud Pública 2011; 28: 691-2.

7. Díaz-Vélez C, Manrique-González LM, Galán-Rodas E, Apolaya-Segura M. Conocimientos, actitudes y prácticas en investigación de los estudiantes de pregrado de facultades de medicina del Perú. Acta Med Peruana 2008; 25: 9-15.

8. Huamaní C, Mayta-Tristán P. Producción científica peruana en medicina y redes de colaboración, análisis del Science Citation Index 2000-2009. Rev Peru Med Exp Salud Pública 2010; 27: 315-25.

9. Taype-Rondán A, Valladares-Garrido D, Eyzaguirre-Villagarcía J, Bendezú-Quispe G, Carbajal-Castro C. Nuevas herramientas para capacitar a los estudiantes de medicina en investigación: experiencias del primer curso virtual de redacción científica de la SOCIMEP. CIMEL 2013; 18: 51-2.

10. Gutiérrez C, Mayta P. Publicación desde el pregrado en Latinoamérica: importancia, limitaciones y alternativas de solución. CIMEL 2003; 8: 54-60. 
11. Maguiña-Vargas C. ¿Por qué investigar en el Perú? Rev Cuerpo Med HNAAA 2013; 6: 6-8.

12. Toro-Huamanchumo CJ. ¿Por qué pertenecer a sociedades científicas en el pregrado de medicina? Rev Cuerpo Med HNAAA 2014; 7: 43-4.

13. Failoc-Rojas VE, Plasencia-Dueñas EA. SOCIEM-UNPRG: impulsando las actividades científicas, académicas y proyección social en estudiantes de medicina. Rev Cuerpo Med HNAAA 2014; $7: 45-6$
14. Nel D, Burman RJ, Hoffman R, Randera-Rees S. The attitudes of medical students to research. S Afr Med J 2014; 104: 32-6.

15. Díaz-Vélez C, León-Jiménez F, Peña-Sánchez R, Soto-Cáceres V, Galán-Rodas E, Málaga-Rodríguez G. Simposio: importancia de la investigación científica en ciencias de la salud. Rev Cuerpo Med HNAAA 2011; 4: 107-14. 